\title{
Uma análise das teleconsultorias assíncronas em saúde auditiva do Núcleo de Telessaúde do Rio Grande do Norte
}

\section{An analysis of asynchronous hearing health teleconsulting at the Rio}

\section{Grande do Norte Telehealth Center}

\author{
Victor Vasconcelos Barros ${ }^{1,2}$ (1), Aryelly Dayane da Silva Nunes ${ }^{1.3}$ (D), \\ Kaio Ramon de Aguiar Lima' (D), Josiane Araújo da Cunha ${ }^{1,4}$ (1), Antônio Higor Freire de Morais ${ }^{1,5}$ (1), \\ Ricardo Alexsandro de Medeiros Valentim ${ }^{1,6}$ (D), Sheila Andreoli Balen $n^{1,2,7}$ (1)
}

\section{RESUMO}

Objetivo: identificar o perfil das teleconsultorias assíncronas na área da saúde auditiva do Núcleo Técnico Científico de Telessaúde do Rio Grande do Norte (RN). Métodos: este estudo é retrospectivo e descritivo. Foram analisados os registros das teleconsultorias de janeiro de 2015 a dezembro de 2019 na plataforma de teleconsultoria do Núcleo de Telessaúde do RN. As teleconsultorias foram filtradas e categorizadas por dois fonoaudiólogos quanto a estado de origem, gênero e profissão do solicitante, tema e objetivo da questão. Resultados: entre as teleconsultorias realizadas no período, foram incluídas, neste estudo, as teleconsultorias na área da saúde auditiva. Em ordem decrescente de frequência, os profissionais solicitantes foram: agentes comunitários de saúde, fonoaudiólogos, agentes de combate a endemias, médicos, enfermeiros, agentes de saúde pública, técnicos e auxiliares de enfermagem e outros. Quanto aos objetivos das teleconsultorias, de maior a menor frequência, foram constatadas perguntas sobre condutas, avaliação, tratamento, relações entre fatores, etiologias, prevenção, sintomas, implantação, acesso ao sistema de saúde e outras. Em relação às temáticas, em ordem descendente, foram observadas perguntas sobre hipoacusia, dispositivos auxiliares de audição, zumbido, otite, programa saúde na escola, emissões otoacústicas, otalgia, labirintite e perfuração timpânica. Conclusão: as teleconsultorias assíncronas sobre saúde auditiva tiveram maior frequência por solicitantes do gênero feminino, agentes comunitários de saúde e fonoaudiólogos, sobre a temática da hipoacusia e de dispositivos auxiliares de audição, com objetivo de tomada de decisões para condutas e avaliação na área da saúde auditiva.

Palavras-chave: Audiologia; Audição; Telemedicina; Teleconsultoria; Atenção primária de saúde

\begin{abstract}
Purpose: To identify the profile of asynchronous hearing health teleconsulting at the Scientific-Technical Center for Telehealth in Rio Grande do Norte (RN). Methods: This study is retrospective and descriptive. Teleconsulting records from January 2015 to December 2019 on the telehealth platform of the Telehealth Center of RN were analyzed. The teleconsultations were filtered and categorized by two speech-language therapists regarding the applicant's state of origin, gender and profession, theme and objective of the question. Results: Among the teleconsultations carried out in the period, the ones regarding the hearing health area were included in this study. In decreasing order of frequency, the requesting professionals were: community health agents, speech-language therapists, endemic disease control agents, doctors, nurses, public health agents, nursing technicians and assistants and others. As for the objectives of teleconsulting, from higher to lower frequency, were identified questions about: conduct, evaluation, treatment, relations among factors, etiologies, prevention, symptoms, implementation, access to the health system and others. Concerning the themes, in descending order, were observed questions about hearing loss, hearing aid devices, tinnitus, otitis, school health program, otoacoustic emissions, otalgia, labyrinthitis and tympanic perforation. Conclusion: Asynchronous teleconsulting on hearing health was more frequent on female applicants, community health agents and speech-language therapists, concerning the theme of hearing loss and hearing aid devices, to make decisions for conducts and evaluation in the health area hearing.
\end{abstract}

Keywords: Audiology; Hearing; Telemedicine; Remote consultation; Primary health care

\footnotetext{
Trabalho realizado na Universidade Federal do Rio Grande do Norte - UFRN - Natal (RN), Brasil.

${ }^{1}$ Laboratório de Inovação Tecnológica em Saúde - LAIS, Universidade Federal do Rio Grande do Norte - UFRN - Natal (RN), Brasil.

${ }^{2}$ Programa Associado de Pós-graduação em Fonoaudiologia, Universidade Federal do Rio Grande do Norte - UFRN - Natal (RN), Brasil.

${ }^{3}$ Programa de Pós-graduação em Saúde Coletiva da Universidade Federal do Rio Grande do Norte - UFRN - Natal (RN), Brasil.

${ }^{4}$ Secretaria de Saúde Pública do Estado do Rio Grande do Norte - SESAP - Natal (RN), Brasil.

${ }^{5}$ Instituto Federal do Rio Grande do Norte - IFRN - Mossoró (RN), Brasil.

${ }^{6}$ Departamento de Engenharia Biomédica, Universidade Federal do Rio Grande do Norte - UFRN - Natal (RN), Brasil.

${ }^{7}$ Departamento de Fonoaudiologia, Universidade Federal do Rio Grande do Norte - UFRN - Natal (RN), Brasil.

Conflito de interesses: Não.

Contribuição dos autores: ADSN, KRAL e SAB foram responsáveis pelo delineamento do estudo; VVB, KRAL e SAB foram responsáveis pela coleta e análise dos dados; Todos contribuíram na redação do manuscrito; VVB, JAC, AHFM, RAMV e SAB foram responsáveis pela revisão da redação final do manuscrito. Financiamento: Fundação Norte Rio Grandense de Pesquisa e Cultura (FUNPEC). N ${ }^{\circ}$ do processo 54/2015.

Autor correspondente: Sheila Andreoli Balen. E-mail: sheila@sheilabalen.com.br

Recebido: Agosto 21, 2020; Aceito: Novembro 30, 2020
} 


\section{INTRODUÇÃO}

O Serviço Único de Saúde (SUS) do Brasil abrange cerca de 211 milhões de habitantes. Desses, 3,5 milhões no estado do Rio Grande do Norte e 4 milhões no estado da Paraíba ${ }^{(1)}$.

O Brasil é um dos poucos países que fornece um serviço público de saúde, sendo acessível à toda população, nativa ou não, ofertando desde a atenção básica à saúde, como avaliação de pressão arterial, até serviços de alta complexidade, como a realização de procedimentos cirúrgicos ${ }^{(2)}$. Contudo, há uma demanda maior que a capacidade de oferta dos serviços ${ }^{(3)}$ e necessidade da presença de reguladores para gerir essa busca por atendimentos, principalmente, de baixa e média complexidade ${ }^{(4)}$.

Tendo em vista as dificuldades de acesso aos serviços de saúde e com o objetivo de promoção da educação permanente de profissionais da saúde, o Ministério da Saúde instituiu o Programa Telessaúde Brasil(5) ${ }^{(5)}$ programa foi criado em razão da possibilidade de fortalecimento e melhoria da qualidade do atendimento da atenção básica no Sistema Único de Saúde, por meio das Tecnologias de Informação e Comunicação (TIC), facilitando a interação entre profissionais da atenção básica com profissionais da atenção especializada. O intuito também foi de diminuir encaminhamentos desnecessários, agilizar o atendimento ao público e, dessa forma, obter melhor custoefetividade. Em 2011, o programa passou a ser chamado de Programa Nacional Telessaúde Brasil Redes, foi ampliado e conta com ações de teleconsultoria, telediagnóstico, telerregulação, telemonitoramento e teleducação ${ }^{(6)}$.

O serviço de teleconsultoria assíncrona que passou a ser disponibilizado pelo Programa Telessaúde Brasil Redes também envolveu a Fonoaudiologia. Atualmente, existem 26 núcleos de Telessaúde em 23 estados vinculados ao programa e, entre estes, há relato da inserção de fonoaudiólogos em seis núcleos: Maranhão (MA), Minas Gerais (MG), Mato Grosso do Sul (MS), Rio Grande do Norte (RN), Rio Grande do Sul (RS) e Santa Catarina (SC) ${ }^{(7)}$.

O Núcleo Técnico Científico de Telessaúde do Rio Grande do Norte está em funcionamento desde $2011^{(8)}$, com inserção de docentes da Universidade do Rio Grande do Norte, fonoaudiólogos, a partir de 2015, por meio da teleconsultoria assíncrona nas áreas da saúde auditiva e da reabilitação auditiva. A partir de 2018 , o programa passou a ter um bolsista, fonoaudiólogo, aluno de mestrado, por 20 horas nas atividades de teleconsultoria na área da saúde auditiva e no desenvolvimento de conteúdos educativos ofertados por meio de webpalestras, acessíveis tanto dentro da plataforma de teleconsultoria, como pelo canal do YouTube do Núcleo de Telessaúde do Rio Grande do Norte.

Os diferentes núcleos no país, em geral, recebem recursos a partir do Programa Telessaúde, para formações para o uso da plataforma com profissionais da atenção básica. A teleconsultoria assíncrona consiste na realização de uma questão por um profissional de saúde, enviada por meio eletrônico para um teleconsultor, podendo tratar de manejo, encaminhamentos, políticas de implantação de serviço, condutas e procedimentos clínicos, ações de saúde e questões relativas ao processo de trabalho na atenção básica. Há profissionais de diversas áreas de formação cadastrados na plataforma, que podem ser telerreguladores ou teleconsultores.

O telerregulador é um profissional de nível superior, com experiência na atenção básica, que analisa e classifica as dúvidas enviadas pelos profissionais dos municípios remotos.
O teleconsultor é um profissional da área da saúde, também de nível superior, que responde às dúvidas dos solicitantes com base em evidências científicas adequadas às características locorregionais e com caráter educacional, em até 72 horas após recebê-las. Esse serviço contribui para evitar ou indicar encaminhamentos dentro das Redes de Atenção à Saúde do SUS. ${ }^{(9)}$

A partir das teleconsultorias, é possível analisar demandas frequentes para auxiliar na formação continuada do profissional da atenção primária à saúde e facilitar a sua busca em fontes confiáveis, com respostas claras e fundamentadas em evidências científicas. Um dos principais objetivos da teleconsultoria é contribuir para a redução na quantidade de encaminhamentos para outros níveis assistenciais, aspecto que foi constatado em estudo que avaliou o impacto do Programa Telessaúde Brasil Redes no estado do Rio Grande do Norte ${ }^{(10)}$. Nesse mesmo estudo, evidenciou-se que o programa foi bem avaliado pelos profissionais de saúde, sendo que, na época do estudo, o programa tinha uma cobertura de $72 \%$ dos municípios do Rio Grande do Norte. Contribuiu para a educação permanente em saúde e constatação de desafios ainda a serem vencidos, como a inclusão digital de muitos profissionais e a falta de estrutura das unidades básicas de saúde, em relação à conectividade e à estrutura física ${ }^{(10,11)}$.

A telessaúde contribui para diminuir as barreiras gerais, frequentemente criadas pela distância, infraestrutura de viagens precárias, mau tempo e distribuição desigual dos prestadores de cuidados de saúde em contextos urbanos e rurais, ou mesmo em regiões do mundo ${ }^{(12)}$. A teleconsultoria é voltada para os profissionais de saúde, em especial os da atenção básica, de nível médio e superior, de forma que estes possam ter apoio de profissionais expertises em diferentes temáticas para os processos de trabalho, o que contribui, de forma efetiva, para a educação dos profissionais de saúde, estreitando os laços e diminuindo as distâncias ${ }^{(13)}$.

Diante do exposto, o objetivo deste estudo foi identificar o perfil das teleconsultorias assíncronas na área da saúde auditiva do Núcleo Técnico Científico de Telessaúde do Rio Grande do Norte.

\section{MÉTODO}

Este é um estudo primário, retrospectivo e descritivo. Foi utilizado o banco de dados da plataforma Telessaúde (http://telessaude.ufrn.br/), a partir do filtro disponível na própria plataforma e identificadas as teleconsultorias realizadas no período de 1 de janeiro de 2015 a 31 de dezembro de 2019. Foram incluídas as teleconsultorias cadastradas na área da saúde auditiva, pelos requisitantes, e que, de fato, estavam dentro dessa temática.

$\mathrm{Na}$ análise dos dados, foram extraídas as seguintes informações das teleconsultorias: local de origem, formação dos profissionais, temáticas abordadas e objetivo das teleconsultorias. Quanto aos locais, foram observadas a origem das teleconsultorias por cidades do Rio Grande do Norte e da Paraíba. No que se refere aos profissionais, foram categorizados em: nível superior (médicos, fonoaudiólogos, fisioterapeutas, nutricionistas, cirurgiões dentista, enfermeiros, assistentes sociais, psicólogos, supervisores administrativos e visitadores sanitários); nível médio (agentes comunitários de saúde, técnicos de enfermagem, agentes de ação social, agentes de combate às endemias, agentes de saúde pública, auxiliares de enfermagem, auxiliares em saúde 
bucal e assistentes sociais); nível básico (agentes comunitários de saúde e agentes de saúde pública).

A análise das temáticas e dos objetivos das teleconsultorias foi realizada por dois fonoaudiólogos, de forma independente. Cada fonoaudiólogo categorizou as temáticas e os objetivos de forma ampla e livre, com base nas questões formuladas pelos profissionais. Em casos de divergência, obtiveram auxílio de um terceiro para desempate, a fim de definir as categorias de temas e objetivos das teleconsultorias.

A partir da obtenção das informações, foi realizada análise descritiva de frequência absoluta e relativa de cada tema e objetivos das teleconsultorias.

\section{RESULTADOS}

O banco de dados da plataforma do Núcleo Técnico Científico de Telessaúde do Rio Grande do Norte contava com 16.272 teleconsultorias assíncronas, recebidas no período de 1 de janeiro de 2015 a 31 de dezembro de 2019, sendo 2.422 devolvidas, em casos de questionamentos incompletos ou inadequados, e 13.850 respondidas e finalizadas. Utilizando o filtro da área de saúde auditiva do banco de dados, foram identificadas 267 teleconsultorias, sendo que 62 foram devolvidas pelo telerregulador, por ausência de informações ou dúvidas, permanecendo 205 teleconsultorias. Destas, foram excluídas 42, pois, embora estivessem cadastradas na área da saúde auditiva, não tinham relação com essa temática. Sendo assim, a amostra deste estudo constou de 163 teleconsultorias assíncronas, representando $1 \%$ das teleconsultorias efetivamente realizadas nesse núcleo.

Os dados obtidos destacaram que a maior parte $(72,39 \%, \mathrm{n}=118)$ das teleconsultorias incluídas foi realizada pelo estado do Rio Grande do Norte, enquanto $24,53 \%(n=40)$ foram originadas na Paraíba. Constataram-se cinco teleconsultorias de origens não registradas.

Com relação ao gênero dos profissionais, $51,53 \%(\mathrm{n}=84)$ eram mulheres. A análise do perfil de profissionais que iniciaram uma teleconsultoria revelou maior frequência de participação dos agentes comunitários de saúde $(36,8 \% \mathrm{n}=60)$, assim como fonoaudiólogos $(15,95 \% \mathrm{n}=26)$ e agentes de combate a endemias $(7,97 \% \mathrm{n}=13)$ (Figura 1).

Quando analisados os níveis de escolaridade dos teleconsultores solicitantes, a maioria $(52,76 \%)$ possuía ensino médio completo, seguido de ensino superior completo $(41,10 \%)$ e, em menor quantidade, usuários com ensino básico $(3,68 \%)$, sendo que $2,45 \%$ dos teleconsultores não descreveram a escolaridade.

Após consenso entre os pesquisadores, foram considerados os seguintes temas: dispositivos auxiliares de audição, emissões otoacústicas, labirintite, otalgia, otite média, perda auditiva, perfuração da membrana timpânica, Programa Saúde na Escola, zumbido e outros. O tema mais frequente foi a perda auditiva $(45,40 \% \mathrm{n}=74)$, seguido por dispositivos auxiliares de audição (aparelho de amplificação sonora individual - AASI e implante coclear - IC) $(12,88 \% \mathrm{n}=21)$ e zumbido $(9,2 \% \mathrm{n}=15)$ (Figura 2$)$.

Com relação aos objetivos, foram caracterizados os seguintes: acesso aos serviços de saúde, avaliação auditiva, conduta, etiologia, informações adicionais, implantação de serviços de saúde, prevenção, relações entre fatores, sintomas e tratamento. O mais ocorrente foi sobre conduta $(31,28 \% \mathrm{n}=51)$, seguido por avaliação $(11,04 \% \mathrm{n}=18)$ e etiologia $(9,2 \% \mathrm{n}=15)$ (Figura 3).

\section{DISCUSSÃO}

A análise dos dados evidenciou que o Rio Grande do Norte foi contemplado com maior número de teleconsultorias realizadas na área da saúde auditiva, quando comparado ao estado da Paraíba, o que pode ter relação com a frequência maior de treinamento nos municípios do Rio Grande do Norte, no período. Ao consultar os registros do Programa de Telessaúde durante o período de 2015 a 2019, constatou-se que foram realizados 68 treinamentos em municípios do Rio Grande do Norte e 28 em municípios da Paraíba. Esse aspecto pode ter relevância em ser apontado, pois demonstra a necessidade de treinamentos prévios para que os profissionais da atenção básica passem a utilizar o sistema para encaminhar suas questões via plataforma de teleconsultoria do Rio Grande do Norte. É por

\section{\% Teleconsultas Enviadas por cada Profissão}

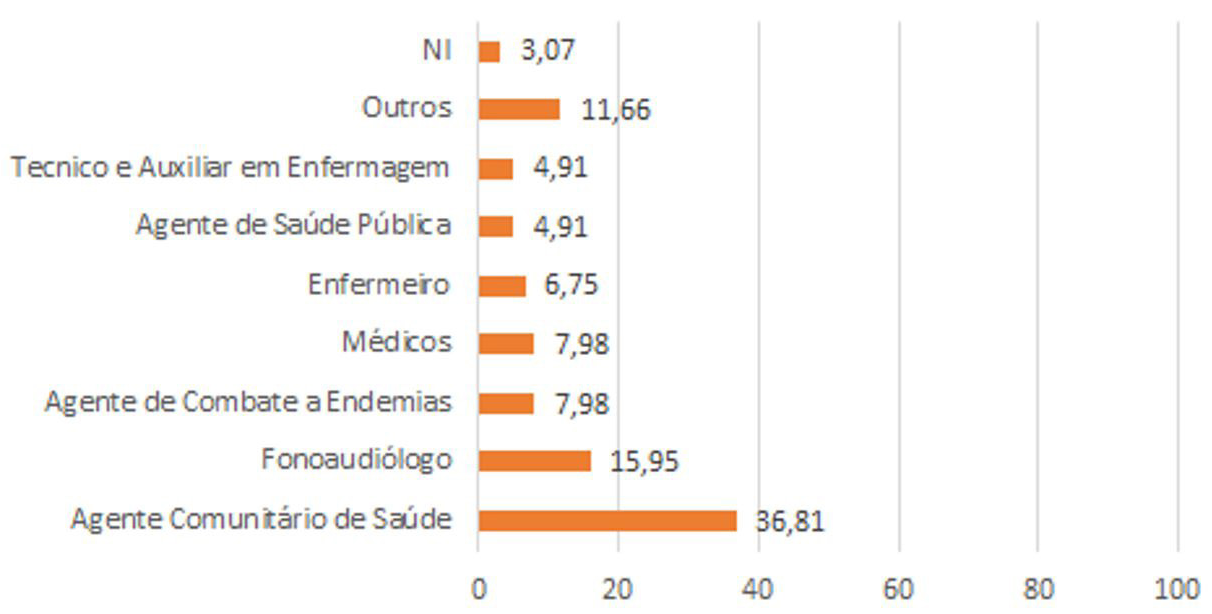

Figura 1. Profissionais solicitantes de teleconsultoria em saúde auditiva no Núcleo de Telessaúde do Rio Grande do Norte (2015-2019) Legenda: \% = percentual; $\mathrm{NI}=$ não informado 


\section{\% Tema da Teleconsultoria}

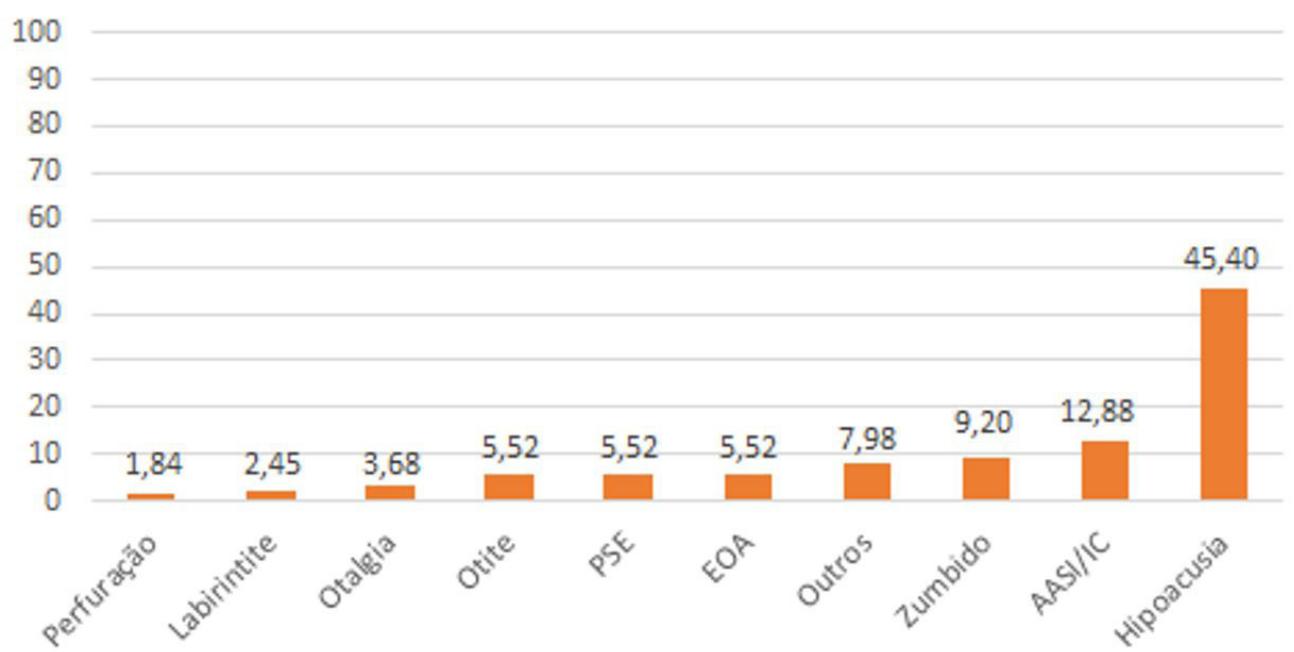

Figura 2. Frequência das temáticas das teleconsultorias

Legenda: \% = percentual; PSE = programa de saúde do escolar; EOA = emissões otoacústicas; $A A S I$ = aparelho de amplificação sonora individual; $I C=$ implante coclear

\section{$\%$ Objetivo da Teleconsultoria}

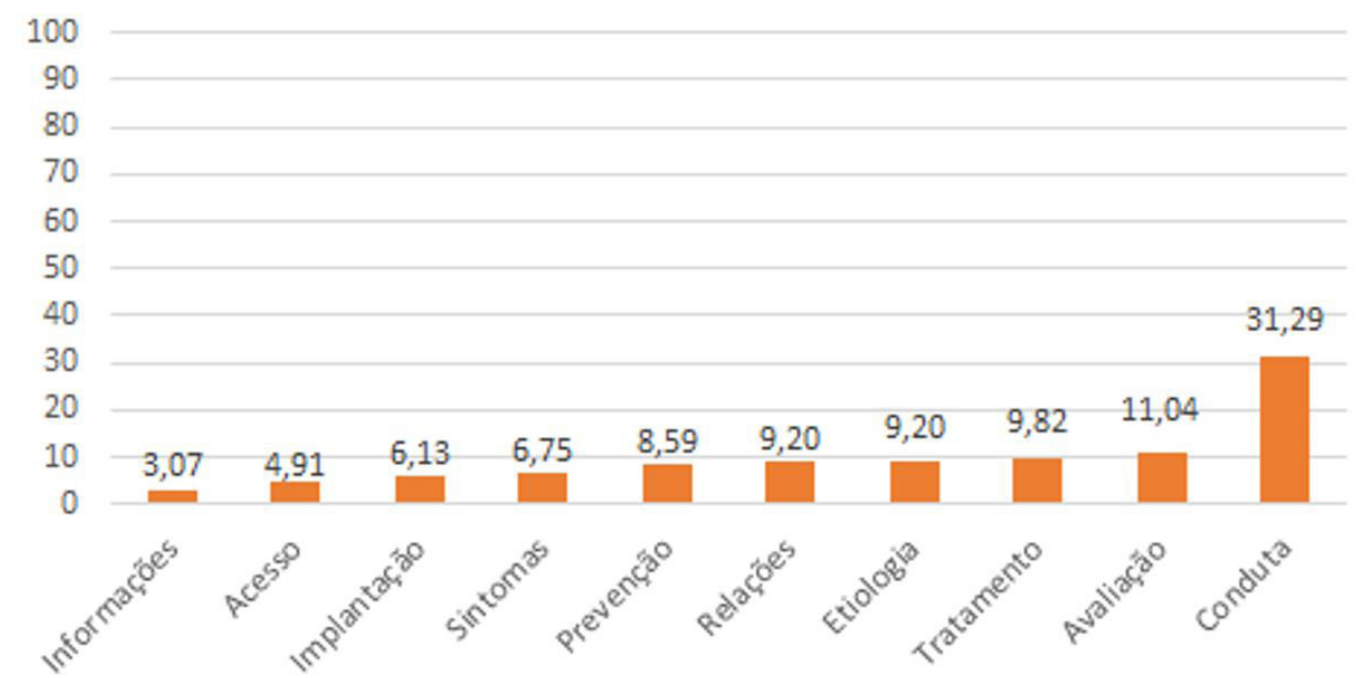

Figura 3. Frequência de ocorrência dos objetivos das teleconsultorias Legenda: $\%=$ percentual

meio desses treinamentos que muitos profissionais tomam conhecimento da existência desse serviço ${ }^{(14)}$.

A relação de maior participação do gênero feminino também se aproximou do perfil de estudo prévio sobre teleconsultorias ${ }^{(15)}$, associando o fato de o gênero feminino ser o mais frequente entre os profissionais ligados à saúde, sendo a Fonoaudiologia uma das profissões compostas, aproximadamente, por $90 \%$ de mulheres ${ }^{(16)}$.

Foi possível observar a participação dos agentes comunitários de saúde como principais profissionais a utilizarem a plataforma para teleconsultorias na saúde auditiva. Esse dado é interessante por estar à frente do número de fonoaudiólogos que realizaram questionamentos e apontados como grupo de maior interesse em outros estudos ${ }^{(17)}$, além de cumprirem um dos principais objetivos do Programa Telessaúde Brasil Redes de facilitar a qualificação de acesso às especialidades, melhorando as tomadas de decisões para encaminhamentos ${ }^{(18)}$.

As vantagens potenciais da telessaúde são particularmente atraentes no campo da atenção à saúde auditiva global, em que há escassez de profissionais de saúde auditiva, capazes de detectarem precocemente a deficiência auditiva.

A maior ocorrência de teleconsultorias terem sido do Estado do Rio Grande do Norte pode ter sido também induzido pelos treinamentos ofertados nos municípios, visto que $70,1 \%$ dos treinamentos realizados pelo núcleo, durante o período estudado, ocorreram no estado do Rio Grande do Norte.

Um dado relevante é que mesmo com menor frequência em comparação aos fonoaudiólogos, os médicos e enfermeiros, além de todos os demais profissionais da saúde, também realizaram perguntas na área da saúde auditiva, informação que reforça 
o conceito de que as teleconsultorias permitem a interação interprofissional de maneira efetiva ${ }^{(19)}$.

Entre os profissionais de nível superior, a maior frequência de fonoaudiólogos (Figura 1) solicitando respostas ao fonoaudiólogo da teleconsultoria evidenciou o anseio destes por formação continuada em determinados pontos da sua prática profissional.

A alta demanda de informações sobre perda auditiva e dispositivos auxiliares de audição demonstrou que, mesmo com políticas públicas consolidadas para saúde auditiva, ainda há baixa divulgação de promoção à saúde auditiva para a população.

O surgimento frequente de teleconsultorias sobre a temática de dispositivos auxiliares de audição (Figura 2) também destacou a importância do acompanhamento contínuo, visto que a retenção de informações e orientações fornecidas aos usuários desses dispositivos, independente do grau de perda auditiva, é reduzida ${ }^{(20)}$.

Considerando-se que o tema mais abordado foi a hipoacusia, retoma-se a importância da realização de capacitações dos agentes de saúde pública, desde a identificação até a conduta ${ }^{(21)}$, sendo a própria teleconsultoria uma ferramenta para acompanhamento continuado dessas capacitações, junto a outros meios de teleducação, como as webpalestras.

Embora a temática do Programa Saúde na Escola não tenha constado entre as mais comentadas, é interessante destacar que $66 \%$ das teleconsultorias sobre o tema envolviam saúde auditiva, mas não foram provenientes de fonoaudiólogos, mostrando a importância do Programa Telessaúde em outros programas multidisciplinares.

Com relação ao principal objetivo das teleconsultorias em saúde auditiva, as condutas se destacaram (Figura 3), revelando a importância da saúde baseada em evidências, para que profissionais, mesmo que iniciantes ou especializados em outras áreas, possam agir com segurança e da forma mais eficaz, do encaminhamento até o processo de reabilitação, evitando, assim, encaminhamentos desnecessários e mantendo os pacientes dentro da atenção básica, quando pertinente ${ }^{(22)}$.

Outro estudo também trouxe a conduta como principal tema abordado nas teleconsultorias, o que é um ótimo indicador da utilização das teleconsultorias por profissionais da área da saúde para a discussão sobre manejo, encaminhamentos, políticas de implantação de serviço, condutas e procedimentos clínicos, ações de saúde e questões relativas ao processo de trabalho na atenção básica ${ }^{(17)}$.

Diferentemente do encontrado, outras pesquisas apontaram maior busca voltada a tratamento e etiologia. Apesar de conduta ainda estar entre os temas mais abordados, apareceu em menor proporção nas teleconsultorias analisadas. A diferença pode ter ocorrido pelo foco de estudo, visto que, em teleconsultorias voltadas à Medicina, há maior procura por tratamento farmacológico ${ }^{(23,24)}$.

Apesar da baixa demanda por informações adicionais, as respostas das teleconsultorias apresentaram indicação de referências e/ou materiais complementares, além da resposta à questão, incentivando a formação continuada entre os profissionais de saúde.

Neste estudo, a proporção de teleconsultorias em saúde auditiva nos núcleos de Telessaúde do Rio Grande do Norte e da Paraíba foi maior, quando comparada a outros estudos, que variaram de $0,2 \%$ a $0,8 \%$ das teleconsultorias dos núcleos estudados ${ }^{(17,25)}$. Essa variação pode se relacionar com uma inserção gradual da Fonoaudiologia na Telessaúde.

Infere-se, portanto, que talvez a pouca demanda das teleconsultorias na área da Fonoaudiologia no Programa Telessaúde Brasil Redes possa ocorrer, ainda, pela falta de conhecimento dos profissionais que atuam na atenção primária à saúde nos municípios, bem como pela própria escassa inserção de fonoaudiólogos nas equipes dos núcleos de Telessaúde em outros estados, atuando como telerreguladores e teleconsultores. Outro aspecto a ser apontado é a não inclusão da temática de Telessaúde no conteúdo das disciplinas curriculares nos cursos de graduação em Fonoaudiologia, embora essa realidade tenha começado a se transformar em razão da necessidade de que os graduandos em Fonoaudiologia possam atender sob supervisão, mesmo que à distância do paciente ${ }^{(26)}$. O Conselho Federal de Fonoaudiologia regulamentou a atividade de telefonoaudiologia, incluindo a teleconsultoria entre as suas práticas oficiais ${ }^{(27)}$

Pôde-se observar, contudo, que ainda há uma demanda inferior de teleconsultorias para a capacidade adequada de oferta, o que indica a necessidade de repensar a agenda profissional para facilitar o acesso dos fonoaudiólogos ao portal de Telessaúde durante o período de atendimento presencial ${ }^{(25)}$. Em outros países, mesmo no serviço de saúde privado, ainda é possível observar dificuldade de adesão às teleconsultorias. É preciso, em futuros estudos, identificar de forma mais aprofundada os motivos para a ocorrência dessa dificuldade ${ }^{(28)}$.

Destaca-se que a Telessaúde tem um importante papel na ampliação do acesso à serviços especializados de saúde, sendo notória a participação e o envolvimento de profissionais de nível básico e médio de escolaridade, a saber os agentes comunitários de saúde. Esses profissionais são de extrema importância para as ações desenvolvidas pelas equipes de Estratégia Saúde da Família (ESF), pois têm relação muito próxima com os usuários dos serviços de saúde em seu território. Dessa forma, as qualificações que recebem por meio das teleconsultorias podem se traduzir em aperfeiçoamento das ações de busca ativa proporcionadas por eles, uma vez que são os que possuem maior contato com a população no território. Assim, fica evidenciada a importância da Telessaúde para encurtar caminhos e estabelecer laços mais fortes entre o serviço de saúde que está na ponta. Com isso, ocorre a qualificação e ampliação do acesso a outras especialidades de forma muito mais rápida e desburocratizada. Tanto o profissional de nível superior pode fazer uso da teleconsultoria para melhoria ou avaliação de sua conduta, como outros profissionais de nível médio e básico podem consultar o serviço para aperfeiçoamento dos cuidados à população.

\section{CONCLUSÃO}

O perfil das teleconsultorias assíncronas na área da saúde auditiva no Programa Telessaúde estudado evidenciou predominância nos municípios do Rio Grande do Norte, em relação à Paraíba. As teleconsultorias foram solicitadas por agentes comunitários de saúde, seguidos por fonoaudiólogos, com maior frequência de profissionais do gênero feminino, sobre a temática da perda auditiva e dos dispositivos auxiliares da audição, relacionadas, predominantemente, a condutas, avaliação e tratamento.

\section{AGRADECIMENTOS}

À Fundação Norte Rio Grandense de Pesquisa e Cultura, pelo apoio concedido para realização da pesquisa e ao Ministério da Saúde, pelo fomento ao Núcleo Técnico Científico de Telessaúde do Rio Grande do Norte. 


\section{REFERÊNCIAS}

1. IBGE: Instituto Brasileiro de Geografia e Estatística. Projeção da população 2020 [Internet]. Rio de Janeiro: IBGE; 2020 [citado 2020 Jul 1]. Disponível em: ttps://www.ibge.gov.br/apps/populacao/projecao/

2. Brasil. Ministério da Saúde. O Sistema Único de Saúde (SUS): estrutura, princípios e como funciona [Internet]. Brasília: Ministério da Saúde; 2020 [citado 2020 Jul 1]. Disponível em: http://www.saude.gov.br/ sistema-unico-de-saude

3. Faria RC, Campos EMS. Demanda espontânea na estratégia de saúde da família: uma análise dos fatores que a influenciam e os desafios na reorientação do modelo assistencial do SUS. Rev Panam Salud Publica. 2012;15(2):148-57.

4. Vieira D, Barbosa S, Barbosa NB, Najberg E. Regulação em Saúde: desafios à governança do SUS Health Regulation: challenges to the Unified Health System governance. Cad Saude Colet. 2016;24(1):4954.

5. Brasil. Ministério da Saúde. Portaria $n^{\circ} 35$, de 4 de Janeiro de 2007. Diário Oficial da União [Internet]; Brasília; 2007 [cited 2020 Ago 21]. Disponível em: http://bvsms.saude.gov.br/bvs/saudelegis/gm/2007/ prt0035_04_01_2007_comp.html

6. Brasil. Ministério da Saúde. Portaria n ${ }^{\circ} 2.546$, de 27 de Outubro de 2011. Redefine e amplia o Programa Telessaúde Brasil, que passa a ser denominado Programa Nacional Telessaúde Brasil Redes (Telessaúde Brasil Redes). Diário Oficial da União [Internet]; Brasília: Ministério da Saúde; 2011 [cited 2020 Ago 21]. Disponível em: https://bvsms. saude.gov.br/bvs/saudelegis/gm/2011/prt2546_27_10_2011.html

7. Saúde BV em. Núcleos do Programa Telessaúde Brasil Redes [Internet]. 2020 [cited 2020 Jun 30]. Disponível em: https://aps.bvs.br/rede-decolaboradores/

8. de Freitas IMA. Impactos do Programa Nacional Telessaúde Brasil Redes na Qualidade dos Serviços de Saúde prestados na atenção básica do Estado do Rio Grande do Norte. Natal: Universidade Federal do Rio Grande do Norte; 2016.

9. Barton KS, Wingerson A, Barzilay JR, Tabor HK. "Before Facebook and before social media... we did not know anybody else that had this": parent perspectives on internet and social media use during the pediatric clinical genetic testing process. J Community Genet. 2019;10(3):375-83. http://dx.doi.org/10.1007/s12687-018-0400-6. PMid:30569339.

10. Freitas IMA, Valentim RAM, Guerra CLB No, Veras NVR, Morais AHF, Hekis HR. O impacto do Programa Telessaúde Brasil Redes na Saúde Pública do Estado do Rio Grande do Norte. In: Secretaria de Educação a Distância - SEDIS-UFRN, editor. O RN à luz da Inovação para o Mundo Natal. Natal: SEDIS-UFRN; 2018. p. 223-53.

11. Harzheim E, Gonc MR, Umpierre RN, Siqueira ACS, Katz N, Agostinho MR, et al. Telehealth in Rio Grande do Sul, Brazil: bridging the gaps. Telemed e-Health. 2016;22(11):1-7.

12. Swanepoel D, Clark JL. Hearing healthcare in remote or resourceconstrained environments. J Laryngol Otol. 2019;133(1):11-7. http:// dx.doi.org/10.1017/S0022215118001159. PMid:30022744.

13. Bernardes ACF, Coimbra LC, Serra HO. Utilização do Programa Telessaúde no Maranhão como ferramenta para apoiar a Educação Permanente em Saúde. Rev Panam Salud Publica. 2018;42(134):1-9. http://dx.doi.org/10.26633/RPSP.2018.134.

14. Vinhal W, Vianna Araújo D, Nunes Aranha R. Histórico da normatização da telessaúde e os impactos da regulação da teleconsultoria na atenção primária em Minas Gerais. 2020;5:58-71. http://dx.doi.org/10.36517/ resdite.v5.n2.2020.a5.

15. Damasceno RF, Caldeira AP. Fatores associados à não utilização da teleconsultoria por médicos da Estratégia Saúde da Família Factors associated with the non-use of telehealth consultancy by physicians of the Family Health Strategy. Cien Saude Colet. 2019;24(8):3089-98. http:// dx.doi.org/10.1590/1413-81232018248.28752017. PMid:31389555.

16. Poz MRD, Pierantoni CR, Girardi S. Trabalho em saúde no Brasil formação, mercado de trabalho e regulação da força de trabalho em saúde no Brasil. Rio de Janeiro: FIOCRUZ; 2013. p. 187-233.

17. Lucena AM, Couto EAB, Garcia VS, Alkmim MBM, Marcolino MS. Teleconsultorias de fonoaudiologia em um serviço público de telessaúde de larga escala Speech, Language and Hearing Sciences teleconsultations in a large public telehealth service. Rev CEFAC. 2016;18(6):1395-403. http://dx.doi.org/10.1590/1982-021620161860816.

18. Maeyama MA, Calvo MCM. A integração do Telessaúde nas Centrais de Regulação: a Teleconsultoria como Mediadora entre a Atenção Básica e a Atenção Especializada. Rev Bras Educ Med. 2018;42(2):63-72. http://dx.doi.org/10.1590/1981-52712015v42n2rb20170125.

19. Curioni CC, Thereza M, Cury F. Potencialidades das atividades em nutrição. J Bras Tele. 2013;2(3):93-7.

20. Geraldo T, Ferrari DV, Bastos BG. Orientação ao usuário de prótese auditiva: retenção da informação Guidance to the user of the hearing aid: retention of information. Arq Int Otorrinolaringol. 2011;15(4):4107. http://dx.doi.org/10.1590/S1809-48722011000400002.

21. Alvarenga KF, Bevilacqua MC, Martinez MANS, Melo TM, Blasca WQ, Taga MFL. Proposta para capacitação de agentes comunitários de saúde em saúde auditiva. Pró-Fono Rev Atualização Científica. 2008;20(3):171-6. http://dx.doi.org/10.1590/S0104-56872008000300006.

22. da Silva MAM, Lopes ÉAS. Conduct of Professionals ' Primary Care After Replies of the Requested Teleconsultings : an initial evaluation Resumen. Lat Am J Telehealth. 2017;4(1):82-7.

23. Marcolino MS, Alkmim MB, Assis TG, Sousa LA, Ribeiro AL. Teleconsultorias no apoio à atenção primária à saúde em municípios remotos no estado de Minas Gerais, Brasil. Rev Panam Salud Publica. 2014;35(5-6):345-52. PMid:25211560.

24. Moreira Lucena A, Alkmim MB, Soares Garcia V, De Araújo Brandão Couto E, Marcolino MS. Speech therapy teleconsultations of a public telehealth service in a developing country. Studies in Health Technology and Informatics. [Internet]. 2015; [cited 2020 Ago 21];216:986. Available from: https://www.scopus.com/inward/record.uri?eid=2s2.0-84952036750\&doi=10.3233\%2F978-1-61499-564-7-986\&par tnerID $=40 \&$ md5 $=$ de899a9f6397d3d1ec8db3ec354e58be

25. Schmitz CAA, Harzheim E. Oferta e utilização de teleconsultorias para Atenção Primária à Saúde no Programa Telessaúde Brasil Redes. Rev Bras Med Fam Comunidade. 2017;12(39):1-11. https://doi. org/10.5712/rbmfc12(39)1453.

26. Fernandes FDM, Lopes-Herrera SA, Perissinoto J, Molini-Avejonas DR, Higuera Amato CA, Tamanaha AC, et al. Uso de telessaúde por alunos de graduação em Fonoaudiologia: possibilidades e perspectivas em tempos de pandemia por COVID-19. CoDAS. 2020;1782(4):3-5. http://dx.doi.org/10.1590/2317-1782/20192020190.

27. Brasil. Conselho Federal de Fonoaudiologia. Resolução $n^{\circ}$. 580, de 20 de agosto de 2020. Dispõe sobre a regulamentação da Telefonoaudiologia e dá outras providências. Diário Oficial da União; Brasília; 25 ago 2020.

28. Ranganathan C, Balaji S, Chain S. Key factors affecting the adoption of telemedicine by ambulatory clinics: insights from a statewide survey. Telemed E-Health. 2018:1-8. 